PUBLICACIÓN ANTICIPADA EN LÍNEA (Versión previa a la diagramación). La Revista Tesis Psicológica informa que este artículo fue evaluado por pares externos y aprobado para su publicación en las fechas que se indican en la siguiente página. Este documento puede ser descargado, citado y distribuido, no obstante, recuerde que en la versión final pueden producirse algunos cambios en el formato o forma.

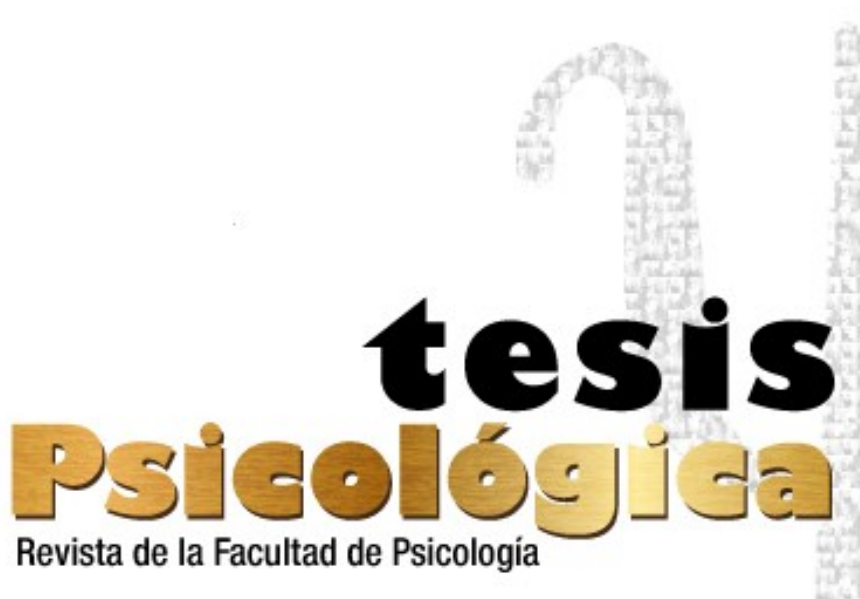




\section{Circulo moral: el caso de los animales no humanos como sujetos de trato moral ${ }^{1}$}

Moral circle: the case of non-human animals as subjects of moral treatment

Camilo O. Moreno-Romero ${ }^{2}$

Recibido: Abril 20 de 2021 Revisado: Mayo 03 de 2021 Aprobado: Junio 04 de 2021

Cómo citar este artículo: Moreno-Romero, C. (2022). Circulo moral: el caso de los animales no humanos como sujetos de trato moral. Tesis Psicológica, 17(1) X-X. https://doi.org/10.37511/tesis.v17n1a1

\section{Resumen}

Antecedentes: El crecimiento y diversificación de las relaciones entre humanos y otras especies animales ha suscitado el interés por la consideración o trato moral hacia dichas especies. Desde la cognición moral, se vienen estudiando tanto las tendencias de razonamiento, como la percepción de capacidades cognitivas que los humanos ven como importantes para considerar a un animal no humano digno de trato moral. Objetivos: El presente artículo realiza una reflexión respecto del denominado círculo moral como espacio psicológico, del cual se incluyen o excluyen agentes desde la percepción de capacidades y creencias asociadas a su estatus moral. Así, se exploran conceptos como el antropocentrismo o especismo, que han sido asociados a la naturaleza de las relaciones entre humanos y otras especies, para profundizar en el conocimiento que se tiene sobre los mismos en el desarrollo infantil. Resultados: La indagación realizada desde la cognición

1Universidad de los Andes - 2020

2Doctor en Psicología - Investigador Posdoctoral Universidad de los Andes Facultad de Educación co.moreno@uniandes.edu.co / ORCID: 0000-0002-5020-615X 
moral en el desarrollo lleva a conocer los antecedentes y cambios en la consideración moral de animales no humanos, además de permitir reflexionar en torno diversas formas de abordar y ampliar el estudio de la inclusión de animales en el círculo moral. Conclusión: Por ello, la parte final del artículo propone acudir a conceptos como la teleología y el desentendimiento moral para seguir profundizando en las bases cognitivas del trato moral a otras especies desde temprana edad, dadas las implicaciones en la educación y la situación ambiental actual.

Palabras clave: Cognición Moral, Capacidades Cognitivas, Círculo Moral, Inclusión Moral, Desarrollo Moral.

\footnotetext{
Abstract

Background: The growth and diversification of the relationships between humans and other animal species has aroused interest in the consideration or moral treatment of these species. From moral cognition, it has been studied tendencies and perception of cognitive capacities that humans see as important to consider a non-human animal worthy of moral consideration. Objectives: From this study, this paper focuses on the so-called moral circle as a psychological space in which agents are included or excluded according to the perception of capacities and beliefs associated with their moral status. Thus, this paper explored concepts associated with the nature of relationships between humans and other species as anthropocentrism or speciesism, deepening into the knowledge of these concepts in child development. Results: The investigation carried out from the moral cognition in development leads to know the antecedents and changes in the moral consideration of nonhuman animals, in addition to allowing reflection on various ways of approaching and expanding the study of the inclusion of animals in the moral circle. Conclusion: For this reason, the final part of this paper proposes to take into account concepts such as teleology and moral disengagement to get deeper knowledge into the cognitive bases of the moral treatment of other species from an early age, given the implications from education and the current environmental situation.
} 
Keywords: Moral Cognition, Cognitive Capacities, Moral Circle, Moral Inclusion, Moral Development.

\section{Introducción}

Las relaciones entre humanos y animales no humanos se han diversificado $\mathrm{y}$ extendido en los últimos años. Por ejemplo, en el 2019 seis de cada 10 hogares colombianos reportaron tener una mascota (Guevara, 2019), ocupando el cuarto lugar en Latinoamérica en este patrón de tendencia creciente (El Tiempo, 2018). Sin embargo y paralelamente, cerca del $80 \%$ de los latinoamericanos tiene una dieta carnívora tradicional (Melo, 2019), y el vegetarianismo en Colombia, aunque creciente, para el 2016 no alcanzaba el 4\% (Climaterra, 2020).

La psicología no es ajena a la mencionada diversificación en las relaciones con otras especies, pues actualmente ésta se interesa por las interacciones e interdependencia con nuestros nichos ecológicos (Uskul \& Oishi, 2020), donde al vínculo que se establece con los animales no humanos se le viene otorgando un papel más activo que pasivo e instrumental en las últimas décadas (Amiot \& Bastian, 2015). Este cambio refleja una visión global y actual, caracterizada por un sentido de conexión psicológica y compromiso más estrecho con diversos seres vivos, que decanta algunas veces en la discusión por el tratamiento y derechos de dichos seres (Amiot \& Bastian, 2017; Auger \& Amiot, 2019; Pinker 2011).

En el núcleo de la discusión está el cuestionamiento sobre los animales ${ }^{3}$ como sujetos de consideración y tratamiento moral. Aunque se concibiera tradicionalmente la moral como un dominio regulador de interacciones entre humanos, que pueden ejercer y juzgar el comportamiento mediante una serie de normas (Heiphetz \& Young, 2014), las relaciones cercanas con otros animales y la

${ }^{3 .}$ En adelante se utiliza varias veces el término animal para referirse a animales no humanos, 
sin dejar de reconocer la naturaleza animal de la especie humana inclusión que se hace de ellos en la vida cotidiana, han llevado a que se discuta el status de estos como agentes y sujetos morales (Clement, 2013; Melson \& Fine, 2010). Es en esta discusión sobre la consideración moral de los animales en la que se centra este artículo que tiene un doble objetivo.

En primer lugar, se pretende reflexionar en torno a los conceptos, capacidades cognitivas y creencias que los humanos tienen en cuenta para considerar un animal como sujeto de consideración moral (Amiot \& Bastian, 2015). Por otra parte, el artículo explora el conocimiento existente frente a dicha consideración moral en el desarrollo infantil, y la manera en que se ha abordado metodológicamente. Las razones para ello, es que son los niños quienes en el futuro tomarán las decisiones en torno a las relaciones con su entorno y los seres vivos que habitan en él (Bone, 2013). Adicionalmente, y como segunda parte, desde temprana edad se establecen relaciones con mascotas y se adquieren hábitos alimenticios particulares, por lo que resulta relevante conocer el surgimiento y curso de las consideraciones establecidas respecto al trato de los animales (Ankomah, 2018). Finalmente, se propone incluir en el estudio de la consideración y estatus moral de los animales conceptos como la teleología (Kelemen,1999), y el desentendimiento moral (Bandura, 2001), que pueden ampliar teórica y metodológicamente el estudio del desarrollo y cambio de la concepción de los animales como sujetos morales.

\section{Inclusión moral: animales y capacidades percibidas}

La consideración o inclusión moral está relacionada con diferentes procesos cognitivos que van desde la percepción de capacidades, hasta una serie de creencias que pueden ser aprendidas en el curso de la vida del individuo. Así, el trato moral a diferentes animales puede incluir desde creencias en la supremacía de los humanos sobre los animales (Dhont \& Hodson, 2014), hasta las capacidades relacionadas con la cognición social, como la toma de perspectiva y la empatía (Berenguer, 2010).

De hecho, estereotipos respecto a seres humanos y su pertenencia a ciertos grupos 
sociales (Sevillano \& Fiske, 2016), e incluso concepciones políticas generales (Dhont, Hodson \& Leite, 2016), inciden en la consideración moral de los animales, haciendo amplio el conjunto de factores a considerar. No obstante, en este texto se tienen en cuenta aquellas capacidades y factores directamente relacionados con el denominado circulo moral, en el que se incluyen aquellas entidades que consideradas dignas de consideración moral dado un conjunto de capacidades o características cognitivas (Crimston, Bain, Hornsey \& Bastian, 2016).

Para comenzar, hay que mencionar que se ha intentado relacionar la inclusión (o exclusión) del circulo moral con tendencias, posturas o modos de razonamiento generales o globales como el antropocentrismo y su contraparte, el ecocentrismo (Kahn, 1996). Una visión o razonamiento antropocéntrico se centraría en los beneficios y la explotación del hombre de su ecosistema en detrimento de los animales, mientras que el ecocentrismo consideraría la naturaleza y los seres vivos como sujetos de trato moral, y los humanos como obligados moralmente a cuidar de estos (Kortenkamp \& Moore, 2001; Waytz, Cacioppo, \& Epley, 2010).

Surgen así varios conceptos asociados. Por ejemplo, el especismo, según el cual la pertenencia o no a una especie determina su estatus moral, otorgando superioridad a los humanos dadas sus capacidades cognitivas (Caviola \& Capraro, 2020). Así, mayor semejanza percibida entre animales y humanos se relaciona con la disposición a ser más solidario con los animales, otorgando un estatus similar al de los humanos a otras especies (Amiot, Sukhanova, Greenaway \& Bastian, 2017). En otro ejemplo relacionado, animales percibidos como más cercanos filogenéticamente fueron elegidos, en comparaciones entre dos especies (por ejemplo, un conejo frente a una tortuga), como más merecedores de compasión, y más susceptibles de empatía (Miralles, Raymond \& Lecointre, 2019).

Es importante mencionar que la solidaridad y la similitud percibida se midieron con pocos ítems y preguntas directas como “¿Son superiores los humanos a los animales?” para el primer caso, y que en cuanto a la empatía solo se preguntaba qué tan dispuesto se estaba a entender los sentimientos de 
determinada especie. Esto no permite conocer qué factores o procesos cognitivos están relacionados con dichas apreciaciones, o si estos inciden directamente en la consideración moral.

Una forma de conocer estos procesos es mediante la relación ente actitudes y creencias frente a los animales y el consumo de carne; por ello, estudios asociados a dicho consumo son parte fundamental de la exploración de la consideración moral hacia otras especies. Por ejemplo, Caviola Everett y Faber (2019) abordan el especismo como constructo psicológico y su relación con la consideración moral, mediante una escala de seis ítems en los que se responde el grado de acuerdo con afirmaciones como "es aceptable tratar animales como posesiones” y “moralmente, los animales valen menos que los humanos"; encontrando asociaciones positivas del especismo con prejuicios como el racismo y con patrones de alimentación basados en la carne. En la misma línea, se encuentra que la orientación de dominancia social (SDO por sus siglas en inglés), la cual se refiere a la superioridad de un grupo sobre los demás y por ello, su llamado a la dominancia y poder sobre otros, predice el especismo, dado que este se basa en la consideración de los humanos como superiores a otras especies (Dhont et al., 2016). De igual manera, el autoritarismo y una tendencia política de derecha (medidas con autorreportes) han mostrado niveles bajos de correlación con actitudes y conductas relacionadas con la consideración moral de otras especies (Timm, 2016).

Sin embargo y más allá de estas tendencias, es necesario profundizar y hacer más concretas aquellas capacidades que los seres humanos perciben como diferenciales, o que nos llevan a concluir que otra especie o ser vivo es digno (o no) de trato moral, pues en términos generales la inclusión o exclusión sería un asunto de categorización (Rosch, 1999). Para el caso del razonamiento moral, la adscripción de estados mentales y capacidades de cognición social juegan un papel clave desde edades tempranas (Cushman, Sheketoff, Wharton \& Carey, 2013), pues el comportamiento es evaluado moralmente de acuerdo con las intenciones, creencias y deseos que subyacen al mismo (Cushman, 2015).

Así, percibir o adscribir estados mentales y emocionales a otro ser se ha visto 
asociado con otorgar estatus moral y tener una actitud hacia la defensa de dichos seres (Rozin, 2007). Sin embargo, este reconocimiento o adscripción riñe con la tendencia a consumir carne animal y su disfrute (Ruby \& Heine, 2011), entrando en una ambivalencia o “paradoja de la carne” (Loughnan, Haslam \& Bastian, 2010), desde la cual se ha estudiado la percepción que los humanos tienen de las capacidades mentalistas en animales.

En este sentido, se ha considerado que estas capacidades pueden incluir, por una parte, capacidades para experimentar emociones y dolor, y por otra, capacidades relacionadas con la agencia como el autocontrol y la planeación de acciones (Waytz, Gray, Epley \& Wegner, 2010). Al pedir que las personas clasifiquen diferentes especies de acuerdo con esta división, se ha encontrado que a mayor percepción de capacidades se juzga más negativamente comer dicho animal (Bastian, Loughnan, Haslam \& Radke, 2012). En hallazgos relacionados, la disposición a consumir carne en el futuro cercano se relaciona con negar las capacidades mentales de especies específicas, disociando la carne del animal de su posible sufrimiento (Bilewicz, Imhoff \& Drogosz, 2011).

En un tipo de acercamiento paralelo a la asignación de estatus moral y capacidades mentales desde la categorización, se encuentra que al presentar un animal directamente como comida o como parte de la dieta de una población, dicho animal se asocia a una menor capacidad de sufrimiento, y por ello, menos susceptible de consideración moral (Bratanova, Loughnan \& Bastian, 2011). De esta manera, la paradoja o disonancia dada por el consumo de carne, parece resolverse al separar animales dignos de trato moral, de aquellos que por patrones de alimentación culturalmente establecidos están categorizados como comestibles, dada la normalidad y naturalidad de esta conducta (Piazza et al., 2015).

Ello lleva a pensar que los humanos asignan estatus moral de manera jerárquica, de acuerdo con las capacidades cognitivas percibidas de otros seres (Brand \& Reina, 2011). Así, un perro es más valorado dado que se considera mentalmente competente y cálido, mientras que a una gallina o una serpiente se les niega el estatus moral dado que no se les asocia con dicha competencia (Dhont, Hodson, 
Loughnan \& Amiot, 2019). Esto se relaciona con la deshumanización y el antropomorfismo, pues la negación de atributos humanos a los animales e incluso otros grupos de personas, los lleva a ser susceptibles de explotación o maltrato sin que esto tenga consecuencias morales (Costello \& Hodson, 2014a; Severson \& Learm, 2016; Grace, 2013).

Sin duda alguna, estas ideas decantan en el ya mencionado y tomado como referente círculo moral, en el cual la organización no es jerárquica sino expansiva pero también basada en la percepción de capacidades y características básicas.

Sin embargo, dicho círculo cambia según ciertas variables y grupos, frente a la jerarquía que se plante más rígida (Bloom, 2010). El estudio referente de este círculo (Crimston et al., 2016) pide ubicar en círculos no únicamente especies animales sino otros seres humanos (familia, amigos) y otros seres vivos como plantas. Lo que se observa es que la expansión, vista como inclusión de más seres en la preocupación por el trato moral, se relaciona con la adscripción de capacidades mentales entendidas como la capacidad de agencia más la capacidad de experimentar dolor.

Mediante esta metodología más completa y de alguna manera gráfica, se ve que familiares y otros seres humanos conocidos están en el primer círculo de consideración, otros seres humanos y mascotas están un poco más alejados, y otras especies y las plantas son aún más lejanos, validando la idea de la presencia de sesgos intergrupo (Dovidio \& Gaetner, 2010). De igual manera, se valida que se considera más importante la vida humana que la vida animal, a excepción de las propias mascotas (Topolski, Weaver, Martin \& McCoy, 2013). Esto se ve corroborado con los hallazgos del moral machine experiment (Awad et al., 2018), el cual, enmarcado en el estudio de las decisiones que un auto manejado por inteligencia artificial debería tomar, encuentra que globalmente se prefiere salvar vidas humanas sobre vidas de animales (desconocidos) en casos de accidentes inevitables.

Para Sevillano y Fiske (2019) las capacidades cognitivas tenidas en cuenta para incluir un ser vivo en el círculo moral se pueden condensar en capacidades de agencia y 
capacidad para experimentar dolor, donde también cobran importancia las emociones como la calidez y ternura evocadas. En este punto, el consumo de carne animal constituye un ejemplo del razonamiento diferencial, con particularidades y ambivalencias respecto a la asignación de estatus moral, desde las justificaciones que se utilizan frente a dicho consumo (Loughnan, Bastian \& Haslam, 2014). Respecto a este, se ha considerado que hay una racionalización o un razonamiento motivado posterior (Mercier, 2011) a la categorización, e incluso al comportamiento que en este caso se da en la relación con animales.

Por ejemplo, aquellos que consumen carne cuando se ven presionados a justificar su consumo, recurren a argumentos religiosos, culturales, o aducen que una dieta carnívora es más masculina aun reconociendo que los vegetarianos pueden ser mejores moralmente, pero al tiempo más débiles en su rol de género (Rothgerber, 2013; Ruby \& Heine, 2011). La necesidad de justificación lleva entonces a validar ideas como "las vacas no sienten dolor”, y a que se apele a motivos incluso de necesidad o naturales (la evolución ha favorecido el consumo de carne animal), o de normalidad basada en las normas sociales asociadas al consumo de animales (Piazza et al., 2015; Piazza \& Loughnan, 2016), resaltando el papel que pueden tener factores culturales y normativos, por una parte, y de formación de hábitos por otra (Bastian \& Loughnan, 2016; Sanbonmatsu, 2014).

El panorama expuesto hasta este punto demuestra que existen una variedad de términos y procesos que se han visto como asociados con la consideración moral hacia los animales. Ya sea que se habla de especismo, antropocentrismo o antropomorfismo, la comunalidad entre estos términos es que apuntan al rol que la percepción de capacidades cognitivas tiene en cómo se trata a otros, en este caso otras especies de animales. Por otra parte, procesos generales como los estereotipos y los sesgos intergrupo, tienen un rol en conductas como el consumo de carne y lo que este consumo implica en las relaciones que establecemos con otros seres vivos. La siguiente sección se centra en abordar lo que se conoce de estos mismos procesos en el desarrollo y en la relación 
de los niños con los animales.

\section{Desarrollo y cambios en la consideración moral de los animales}

El estudio de las relaciones con animales desde temprana edad pareció tener en principio una base instrumental, reflejando valores y actitudes de superioridad basadas en el beneficio humano en dichas relaciones (Duhn, 2012). Sin embargo, ya sea por entender las bases ontogénicas de estas actitudes, o por intentar dar un giro hacia un rol educativo respecto de los animales y el cuidado del ambiente, el trato y consideración de los animales como sujetos de derechos hace parte de discusiones desde diferentes ámbitos (Ankomah, 2018).

Dentro de la psicología, y con especial énfasis en el tema del trato y consideración moral, se encuentran estudios pioneros respecto a la edad en la cual se evidencia razonamiento antropocéntrico o ecocéntrico en niños, no específicamente respecto a los animales, sino al ambiente en general (Kahn, 1996; Kahn, 1997a). Tomando como referentes teorías del desarrollo moral (Piaget, 1965; Nucci \& Turiel,2009), y mediante entrevistas semiestructuradas sobre distintos problemas ambientales relevantes en diferentes entornos, se encuentra que entre los siete y ocho años el razonamiento antropocéntrico predomina, mientras que el razonamiento ecocéntrico, se evidencia más tarde, donde se desaprueba cualquier daño a la naturaleza (Kahn \& Lourenço, 2002). Paralelamente, se plantean conceptos como la biofilia sea por razones benéficas para los humanos o por una conexión con la naturaleza, hay una tendencia o preocupación general y temprana por el trato que se le da a los distintos seres que la componen (Kahn, 1997b; Khan, Severson \& Ruckert, 2009).

En la misma línea, pero ya con un mayor énfasis en animales, Melson (2013) habla de un razonamiento eco-moral que tiene un curso de desarrollo caracterizado por juicios cada vez más complejos respecto al tratamiento que se les da a formas de vida no humanas. En este sentido, hacia la preadolescencia el juicio moral respecto al trato de los animales se da por sus características 
intrínsecas que los lleva a ser valiosos por sí mismos y no por los beneficios que les representan a los humanos (Melson et al, 2009); sin embargo, especies que pueden ser consideradas peligrosas o que generen emociones negativas pueden ser dejadas fuera del razonamiento ecocéntrico (Melson \& Fine, 2010).

Estudios posteriores encuentran que el razonamiento ecocéntrico puede estar presente desde los siete u ocho años en mayor medida que el antropocéntrico, esto si se han tenido experiencias positivas en la interacción con animales (Almeida, Vasconcelos, Strecht-Ribeiro \& Torres, 2013). De igual manera, se ha evidenciado que desde los seis años se justifica un juicio negativo de un comportamiento dañino al ambiente por el daño que este haría a otras especies que se consideran valiosas (Šorytė \& Vilmantė Pakalniškienė, 2019), revelando una preocupación temprana por el trato que se les da a los animales.

Como se ha mencionado anteriormente, el estudio de estas tendencias de razonamiento general se puede ver enriquecido por la búsqueda de asociaciones de dichas tendencias con la percepción de capacidades cognitivas específicas en otros seres. En este sentido, empleando dilemas morales en los cuales se hacía daño a especies hipotéticas (no reales) que variaban en sus capacidades cognitivas, se ha encontrado que niños de nueve años juzgan peor transgredir a una especie que tiene la capacidad de experimentar dolor, por encima de la capacidad para percibir y pensar (Olthof et al., 2008).

En hallazgos relacionados, mediante entrevistas respecto a especies reales en peligro en el entorno de niños entre siete y 10 años, se encuentra que a mayor edad predomina el razonamiento ecocéntrico centrado en las necesidades de los animales, justificado mediante la capacidad de estos para sentir (Ruckert, 2016). En el mismo sentido, niños entre los seis y siete años, aunque consumidores de carne, han expresado que esta es una conducta problemática porque el animal podría sufrir dolor y porque tiene necesidades biológicas que no se cumplirían (Hussar \& Harris, 2010). Esto confirmaría que la agencia y capacidad para experimentar dolor es lo que lleva a la consideración moral y no solo convencional de las relaciones con los animales (Hussar \& Horvat, 2011).

Dados los hallazgos que se vienen mencionando, la clasificación y la idea de círculo 
moral cobra relevancia en el desarrollo. Así, al pedir a niños entre los ocho y los 10 años una clasificación de los animales que más les gustan, se encuentran que mamíferos grandes y pájaros son los preferidos, y que hay una asociación moderada entre este gusto y el razonamiento de que estas especies se deben salvar en caso de estar en peligro (Almeida et al, 2013). Esto se relaciona con la preferencia y compasión por especies filogenéticamente cercanas (Miralles et al., 2019), aunque el gusto o preferencia no tiene por qué estar directamente implicado con la inclusión moral.

Cuando se explora específicamente la inclusión moral, se encuentra que la adscripción de vida mental (entendida como capacidad para sentir, actuar con propósito y entender ciertas acciones), resulta asociada positivamente a dicha inclusión en niños entre los cuatro y 10 años, quedando excluidos objetos inanimados y muchas veces robots (Sommer et al., 2019). Acudiendo a la metodología ya mencionada de clasificación en círculos (Crimston et al., 2016), se encuentra que a menor edad (cuatro o cinco años) se incluyen en el círculo más cercano familiares, amigos y mascotas, mientras que sobre los 10 años se incluyen otros animales salvajes y otras entidades, aunque en círculos más lejanos que denotan menos preocupación (Nelder, Crimston, Wilks, Redshaw \& Nielsen, 2018).

Por otra parte, la tendencia vista en adultos a privilegiar la vida humana ante la vida de animales en elecciones dicotómicas para salvar vidas (Awad et al., 2018), se manifiesta de manera más débil en niños. En una muestra de 249 niños entre los cinco y nueve años, 63\% de ellos decían no poder decidir si salvar a un humano frente a un perro, mientras el 35\% reportaba preferir salvar la vida del humano; más importante aún, esta preferencia no estuvo influenciada por la inteligencia y capacidad de sentir adscrita a humanos, perros o cerdos. Esto sugiere que la visión en la cual los humanos son moralmente superiores, y por ello, más importantes en cuanto a

salvar sus vidas, aparece tarde en el desarrollo y se puede ver influenciada por factores sociales o culturales (Wilks, Caviola, Kahane \& Bloom, 2021).

Por último, otro factor asociado a la consideración moral de animales en el 
desarrollo parece ser el surgimiento temprano de la deshumanización. Niños entre los seis y 10 años atribuyeron menor capacidad emocional en términos de felicidad, tristeza y culpa a animales, y mostraron acuerdo ante afirmaciones que establecían que los humanos eran muy diferentes de los animales (Costello \& Hodson, 2014b); lo que a su vez se relacionó con sesgos intergrupo y la lealtad hacia grupos de pertenencia como la familia e incluso las mascotas (Buttlemann \& Böhm, 2014).

Al igual que se expuso en la primera sección, la percepción de capacidades cognitivas asociadas a la agencia, inteligencia, y capacidad para experimentar dolor parecieran jugar un rol fundamental en la consideración moral de los animales desde edades tempranas. Así mismo, tendencias generales como el razonamiento antropocéntrico y ecocéntrico han hecho parte del estudio de dicha consideración, sin embargo, este abordaje se ha enfocado en el medioambiente y no específicamente en otras especies animales. Al mismo tiempo, este estudio no parece poseer un conjunto unificado de términos o constructos, pues en algunos casos se pregunta a los menores por inteligencia, otros por agencia o autonomía, y la capacidad para experimentar dolor algunas veces se trata de manera más general como capacidad para sentir.

Por otra parte, las metodologías utilizadas, aunque adaptadas de los estudios con adultos, se reducen a explorar cuestiones relacionadas con el daño o con la preferencia por ciertas especies. Retomando lo anterior, la siguiente sección reflexiona en torno a algunos aspectos que podrían mejorar los acercamientos presentados desde la cognición moral a las relaciones con animales. Posteriormente, se propone complementar dichos acercamientos con la exploración de constructos como la teleología y el desentendimiento moral en el desarrollo.

\section{Inclusión moral y desarrollo: nuevas posibilidades}

El estudio de la cognición moral se caracteriza por el interés en los estados mentales y capacidades cognitivas que se tienen en cuenta para evaluar y juzgar el comportamiento de otros (Heiphetz \& Young, 2014), y la inclusión o exclusión de animales como sujetos de trato moral no es ajena a este interés. 
Metodológicamente, esta orientación en el estudio de la cognición moral ha llevado a centrarse en elementos como dilemas que ponen en conflicto deberes morales, muchas veces centrado en salvar vidas o evitar accidentes (Christensen, Flexas, Clabrese, Gut \& Gomila, 2014; Awad et al., 2018).

Si bien el estudio de la cognición moral a través de dichos dilemas ha sido importante en cuanto al rol de los estados mentales en el juicio moral desde edades tempranas (Cushman et al., 2013), deja por fuera ciertos elementos importantes en el caso de la consideración moral de los animales. Uno de estos elementos es el contexto, pues como apuntan Hester y Gray (2020), en su mayoría los dilemas o situaciones se han postulado genéricamente, sin hacer distinciones de la identidad de quién juzga o de quién es juzgado desde aspectos culturales o sociales importantes. Esto se puede ver en estudios donde se comparan especies respecto a la compasión o empatía suscitada, sin tener en cuenta la relevancia, presencia o experiencia general en determinadas culturas con dichas especies (Miralles et al., 2019; Amiot et al., 2017).

En los estudios mencionados relacionados con el consumo de carne, se tienen en cuenta aspectos culturales como la orientación política o social general de quienes emiten juicios o creencias (Piazza et al., 2015), pero en el estudio de la consideración moral en el desarrollo, variables culturales y sociales de los niños participantes no son tenidas en cuenta, dificultando conocer los antecedentes o motivadores de sus actitudes y creencias frente a los animales (Melson 2013). Por ejemplo, para Sambonmatsu (2014), ni las dietas carnívoras ni las actitudes frente a los derechos de los animales se desarrollan en el vacío, sino en narrativas culturales y sociales a las que los niños se ven expuestos desde tempranas edades, por lo cual acercamientos al desarrollo desde las influencias sociales, familiares y culturales en general (Overton, 2013) podrían ser más fructíferos.

En este sentido, Schein (2020) propone que la contextualización del estudio de la cognición moral debe pasar no solo por dotar de identidad a quienes juzgan y a quienes son juzgados, sino por la indagación de los valores que guían dicha cognición moral y cómo se han formado. Así, la prioridad que se da a los animales y a ciertas especies puede variar 
entre culturas o incluso intra culturalmente.

Como muestra de ello, se ha visto que, aunque la familiaridad con ciertas especies no cambia las actitudes de los niños frente al trato de las mismas, el afecto o empatía por animales de compañía y empleados en entornos terapéuticos se generaliza a la especie de dichos animales (Fonseca et al., 2016).

Junto con esta reflexión respecto a la necesidad de dar mayor contexto y especificidad a las situaciones empleadas, es importante volver sobre la diversidad de términos usados para referirse a las capacidades cognitivas de los animales (Dhont et al., 2019), puestas impactan directamente las metodologías utilizadas. Se utilizan algunas veces términos amplios como pensar y percibir (Olthof et al., 2008) sin detenerse en cómo conceptualizan los menores estas capacidades, o qué capacidades específicas implica un término como pensar. De igual manera, se utilizan mediciones sobre el antropomorfismo (Severson \& Lemm, 2016) desde capacidades que se enuncian como planeación, agencia y entendimiento (Sommer et al., 2019), sin saber si estas definiciones son equivalentes en diferentes estudios y con diferentes participantes.

Relacionado con lo anterior, se encuentra el hecho de que los estudios se enfoquen en la dimensión moral del daño físico ya sea de extinción, caza y muerte de los animales (Dhont \& Hodson, 2014a), y por ello en la noción de daño, desconociendo que aspectos como la calidez, cooperación y solidaridad también están asociados a la moral (Hester \& Gray, 2020) y se desarrollan desde edades tempranas (Zhang, Grocke \& Tomasello, 2019). Adicionalmente, las preguntas parecen centrarse en la aceptabilidad de ciertos comportamientos, incluso algunas veces preguntando por el cuidado hacia otros (Nelder et al., 2018) que puede no corresponder exactamente con la noción de evitar un daño o de no herir que predomina en la cognición moral. Igualmente, los estudios en cognición moral suelen preguntar por las creencias sobre el castigo o sanción que merece una conducta (Smetana, Jambon, Conry-Murray \& Sturge-Apple, 2012), aspecto que no se ha explorado en los estudios revisados desde la óptica de qué sanciones creen las personas y en especial los niños que merecería el maltrato a los animales. 
Finalmente, y como preámbulo a la reflexión en torno a nuevos conceptos que se pueden incluir en el estudio de la inclusión moral, hay que mencionar que pocos estudios utilizan las justificaciones que dan las personas para responder a las diferentes preguntas en torno al trato moral de los animales. Por ejemplo, algunos estudios las han usado para clasificar el razonamiento como antropocéntrico o ecocéntrico (Kahn,1996; Ruckert, 2016), pero el valor empírico de estas puede ser ampliado al permitir conocer creencias, normas y valores que se expresan cuando se piden razones o explicaciones de una decisión (Malle, 2011). Así, recogiendo tanto los avances expresados a lo largo del texto respecto de la concepción de círculo moral y la inclusión de los animales en este, como las reflexiones hechas sobre algunas debilidades de estos estudios se proponen a continuación la teleología (Kelemen, 1999) y el desentendimiento moral (Bandura, 2004) como constructos que pueden contribuir teórica y metodológicamente al conocimiento de la inclusión y trato moral de los animales.

\section{Teleología como explicación relacionada}

La explicación que se le dan a diversos fenómenos de orden físico, biológico y psicológico es de gran interés para la cognición causal (Legare \& Lombrozo, 2014). Dichas explicaciones surgen y cambian con el desarrollo y en diferentes dominios: así las intenciones explicarían el comportamiento humano, mientras que virus y bacterias se usarían en la explicación de enfermedades (Sloman \& Lagnado, 2015). Es en este punto donde surge la teleología como la disposición a dar explicaciones desde los propósitos, metas de los agentes y, ante todo, funciones de lo que se está explicando (Lombrozo \& Carey, 2006).

Aunque los niños desarrollan explicaciones apropiadas desde la biología para el comportamiento de sus mascotas y de los seres vivos (Geerdts, Van de Walle, \& LoBue, 2015), se ha observado lo que se denomina "promiscuidad teleológica” dado que niños en edad escolar extienden este tipo de explicaciones a diversos seres vivos y fenómenos naturales. Por ejemplo, en vez de decir que no saben o que la 
pregunta es incorrecta ante el cuestionamiento ¿para qué es...? los niños dicen que los leones son para ir al zoológico o las plantas para dar sombra (Kelemen,1999; 2004).

Así, se adjudica la aparición u origen de diversas entidades con el diseño hecho por seres dotados de intencionalidad; es allí donde se puede proponer que esta tendencia o postura explicativa se relacione con el razonamiento antropocéntrico que se ha explorado en este texto como relacionado con la exclusión y negación de tratamiento moral de los animales (Waytz, Cacioppo, \& Epley, 2010; Kahn \& Lourenço, 2002), pues al estar centrada en el propósito o función puede correlacionarse con la tendencia a ver a los animales al servicio de los humanos o como necesarios para su consumo y beneficio general.

De hecho, entre niños de cinco a siete años se ha encontrado la coexistencia de explicaciones biológicas y teleológicas, donde se favorece la actuación de seres superiores o agentes intencionales sobre hechos como la enfermedad o muerte de otras personas y de mascotas (Banerjee \& Bloom, 2015; Busch, Watson-Jones \& Legare, 2017). Así, la promiscuidad teleológica podría estar a la base de las visiones vistas del consumo de carne como algo natural y necesario (Bratanova et al., 2011; Piazza \& Loughnan, 2016), pues estas visiones basadas en el diseño y funcionalidad otorgada por seres superiores podrían afectar estereotipos o categorías sociales (Rhodes, Leslie \& Tworek, 2014), en las cuales de nuevo los animales quedarían excluidos de trato moral, dada la falta de características esenciales como la agencia o inteligencia.

Al mismo tiempo, el hecho de que se haya evidenciado que esta postura disminuye para fenómenos naturales hacia los 10 años (DiYanni \& Kelemen, 2005) hace interesante explorar su curso de desarrollo e influencias tanto en su aparición como disminución respecto a la explicación y categorización animal, pues la tendencia al razonamiento teleológico podría ser adquirida y potenciada a

través del lenguaje y transmisión cultural en general (Harris, 2012; Harris \& Koenig, 2006). Por ello, incluir variables relacionadas con la exposición de niños a educación científica, contacto con animales y narrativas que encuentran en su vida cotidiana sobre 
otros seres, pueden estar relacionadas en las concepciones que expresan sobre el trato a animales y las capacidades de estos respecto a los humanos (Ankhoma, 2018).

\section{Desentendimiento moral y justificaciones}

Al igual que en el terreno de la explicación causal de fenómenos como el comportamiento de distintos seres, en la cognición moral los seres humanos intentan explicar lo coherente o incoherente de su conducta respecto a normas y estándares morales particulares. Surgen así las justificaciones como evidencia del razonamiento moral, y dentro de estas el denominado desentendimiento moral (Bandura, 2004). El desentendimiento se evidencia a partir de una serie de justificaciones útiles para reinterpretar la conducta considerada inmoral, obscurecer el rol causal en ella, y evitar la autocensura (Bandura, Barbaranelli, Caprara \& Pastorelli, 1996). Dichas justificaciones incluyen culpar a la víctima, negar el rol causal o la intencionalidad en la conducta, o minimizar sus consecuencias, evidenciadas estas justificaciones usualmente a través de autorreportes (Gini, Pozzoli \& Hymel, 2014; Moore, 2015; Pozzolli, Gini \& Vieno, 2012).

De esta manera, para el caso de la consideración moral de animales, cuando se den comportamientos que puedan ser considerados inmorales se puede recurrir a excusas como “no sabía que maltrataban a los animales para producir alimentos” (obscurecer responsabilidad), o “no es tan grave si los humanos se benefician” (minimización de consecuencias). Algunos de los estudios mencionados acuden a las justificaciones como respaldo de la existencia de la disonancia cognitiva frente a dietas carnívoras (Ruby \& Heine, 2011; Piazza \& Loughnan, 2016), sin embargo, acercarse desde el desentendimiento proporciona ciertas ventajas diferenciales.

En primer lugar, permitiría clasificar y perfilar de manera más adecuada y sistemática las justificaciones frente a conductas que implican otras especies, abarcando constructos como la deshumanización que también es incluida en el desentendimiento (Bastian \& Haslam, 2010), y que se ha visto como estrechamente relacionado con la exclusión moral desde las capacidades de otros individuos (Costello \& Hodson, 2014b; 
Timm, 2016). Segundo, se ha evidenciado que el conjunto de justificaciones entendidas como desentendimiento moral alcanzan su punto máximo en la adolescencia, aunque su surgimiento y desarrollo requieren clarificación (Oberman, 2013), por lo cual resulta relevante estudiar las variables asociadas al cambio y evolución de estas justificaciones.

Adicionalmente, y relacionado con la necesidad de estudiar aspectos culturales que inciden en la consideración moral de los animales, el desentendimiento está más cercano a la naturaleza social y regulatoria del juicio moral desde las excusas y justificaciones, que, compartidas por un grupo, permiten condenar o aceptar diversos comportamientos (JanoffBulman \& Carnes, 2013). Desde este marco, se puede estudiar cuáles excusas o justificaciones son socialmente aceptadas (y cuáles no), evitando o llevando al castigo social en cuanto a la consideración moral de los animales. Estas excusas surgen desde edades tempranas y se dan en la interacción con pares y con adultos, convirtiéndose la exploración de justificaciones espontáneas en alternativa a los autorreportes (Malle 2011; Moreno-Romero, 2020; Malle, Guglielmo \& Monroe, 2014).

\section{Consideraciones Finales}

El recorrido hecho hasta este punto permite algunas reflexiones respecto al estudio de las relaciones entre humanos y otras especies de animales desde la cognición moral. La diversidad de constructos y métodos mencionado habla principalmente de la importancia que viene cobrando el tema en la psicología cognitiva (Amiot \& Bastian, 2015), y que parece responder al interés social y cambios culturales en las relaciones con animales (Sanbonmatsu, 2014). Se puede decir que la consideración moral de animales se ha abordado ya sea desde

la naturaleza de las relaciones que establecemos con ellos, y desde la percepción que tenemos de sus capacidades cognitivas como sujetos morales. Sin embargo, pareciera que hay una influencia reciproca entre estas visiones y que un acercamiento conjunto resultaría más fructífero en términos investigativos (Grace, 2013).

Esto quiere decir que tanto los cambios en las relaciones de los humanos con otras 
especies, ya sea por mayor cercanía con mascotas, por el conocimiento sobre trato y las consecuencias sobre consumo de otras, incide en cómo respondemos moralmente a nuestro comportamiento y concepción de los animales; así como la percepción de sus capacidades cognitivas incide en la relación diferencial que se establece con ciertas especies. A partir de esto, es ventajoso hablar del círculo moral como el espacio psicológico en el cual se incluyen o excluyen agentes, dependiendo la percepción y razonamiento sobre sus capacidades (Crimston et al., 2016).

Sin embargo, este acercamiento debe ser complementado por variables de aprendizaje social y cultural que puedan incidir en la consideración moral y que dependiendo de experiencias tempranas pueden incidir en la percepción de dichas capacidades (Ankomah, 2018). Es en este punto donde la teleología y el desentendimiento moral pueden ser complementos desde la explicación causal centrado en capacidades cognitivas en el primer caso, y de razonamiento y justificación del comportamiento que puede ser juzgado moralmente desde el segundo.

En la misma línea, la reflexión realizada en este texto invita a pensar en estrategias teóricas y metodológicas que, teniendo en cuenta lo visto en cuanto a la cognición moral y el desarrollo de la misma, sean capaces de informar sobre los antecedentes y las variables no únicamente intraindividuales sino contextuales y ecológicas que influyan en la consideración moral de especies no humanas. En este sentido, los modelos socioecológicos del desarrollo ofrecen una alternativa viable al momento de incluir en la investigación variables contextuales en diferentes niveles y contextos como el familiar, escolar y político (Overton, 2013).

Al tener en cuenta estos aspectos contextuales, el estudio de la consideración moral de animales no humanos a lo largo del desarrollo se verá influenciado, ya sea a manera de mediación o de moderación en términos estadísticos, por la publicidad, las leyes, políticas y normas sociales (Townsend et al., 2020), que en este caso se refieran al trato con animales y otros seres vivos en un entorno particular, y que vayan cambiando con el tiempo, como se refleja en los cambios mencionados en el comportamiento respecto al cuidado de mascotas 
y el consumo de carne animal.

Como se dijo al principio, son los menores quienes tomarán decisiones e interactuarán con otras especies de animales de acuerdo con los estándares morales que establezcan desde ahora (Bone, 2013). Por lo anterior, el conocimiento de los procesos y variables asociadas con la consideración moral de los animales tiene implicaciones educativas fundamentales. Primero porque clarifica el rol que el contacto temprano con animales pueda tener en su trato posterior y en cómo se razona frente a sus derechos. Segundo, porque permite entender la manera en que, en el caso de existir sesgos y tendencias desde la categorización de grupos sociales y especies animales (Dovidio \& Gaetner, 2010), estos puedan ser abordados e incluidos en una conversación crítica sobre el rol de los animales en nuestra sociedad.

Es evidente entonces que en una sociedad que tiene como uno de sus principales desafíos la conservación de la biodiversidad y el enfrentamiento a las consecuencias del cambio climático, las preguntas y respuestas respecto al razonamiento y comportamiento moral frente a diversos seres vivos es preponderante. Por ello, conocer el desarrollo y cambio a lo largo del ciclo vital de las concepciones referentes tanto al medio ambiente como a los seres que lo habitan, debe ocupar un lugar centrar en la psicología del desarrollo y la cognición moral.

\section{Referencias}

Almeida, A., Vasconcelos, C. M., Strecht-Ribeiro, O., \& Torres, J. (2013). Nonanthropocentric Reasoning in Children: Its incidence when they are confronted with ecological dilemmas. International Journal of Science Education, 35(2), 312-334. https://doi.org/10.1080/09500693.2011.608387

Amiot, C. E., \& Bastian, B. (2015). Toward a psychology of human-animal relations. Psychological Bulletin, 141(1), 6-47. https://psycnet.apa.org/doi/10.1037/a0038147

Amiot, C. E., \& Bastian, B. (2017). Solidarity with animals: Assessing a relevant 
Tesis Psicológica | Vol. 17(1) enero-junio /22 | pp. 1-31 | E- ISSN: 2422-045

dimension of social identification with animals. PLoS ONE, 12. https://doi.org/10.1371/journal.pone.0168184

Amiot, C. E., Sukhanova, K., Greenaway K. H., \& Bastian B. (2017) Does HumanAnimal Similarity Lower the Need to Affirm Humans' Superiority Relative to Animals? A Social Psychological Viewpoint, Anthrozoös, 30:3, 499-516, https://doi.org/10.1080/08927936.2017.1335117

Ankomah, W. S. (2018). Animal studies: Let's talk about animal welfare and liberation issues in childhood. Society and Animals, 28(3), 311-326. https://doi.org/10.1163/15685306-12341565

Auger, B., \& Amiot, C. E. (2019). Testing the roles of intergroup anxiety and inclusion of animals in the self as mechanisms that underpin the "pets as ambassadors” effect. Anthrozoos, 32, 5-21. https://doi.org/10.1080/08927936.2019.1550277

Awad, E., Dsouza, S., Kim, R., Schulz, J., Henrich, J., Shariff, A., Rahwan, I. (2018). The Moral Machine experiment. Nature, 563(7729), 59-64. https://doi.org/10.1038/s41586-018-0637-6

Bandura, A. (2001). SOCIAL COGNITIVE T HEORY: An Agentic Perspective. Annual Review of Psychology, 52(1), 1-26. http://doi.org/10.1146/annurev.psych.52.1.1 n

Bandura, A. (2004). Selective exercise of moral agency. In H. J. Thorkildsen, T.A \& Walberg (Ed.), Nurturing Morality (1st ed., pp. 37-57). Boston: Kluwer Academic.

Bandura, A., Barbaranelli, C., Caprara, G. V., \& Pastorelli, C. (1996). Mechanisms of moral disengagement in the exercise of moral agency. Journal of personality and social psychology, 71(2), 364. http://dx.doi.org/10.1037/0022- 3514.71.2.364

Banerjee, K., \& Bloom, P. (2015). "Everything happens for a reason": children's beliefs about purpose in life events. Child development, 86(2), 503-518. https://doi.org/10.1111/cdev.12312

Bastian, B., \& Haslam, N. (2010). Excluded from humanity: The dehumanizing effects of social ostracism. Journal of Experimental Social Psychology, 46(1), 107-113. 
Tesis Psicológica | Vol. 17(1) enero-junio /22 | pp. 1-31 | E- ISSN: 2422-045

https://doi.org/10.1016/j.jesp.2009.06.022_

Bastian, B., \& Loughnan, S. (2017). Resolving the Meat-Paradox: A Motivational Account of Morally Troublesome Behavior and Its Maintenance. Personality and Social Psychology Review, 218), 299. https://doi.org/10.1177/1088868316647562

Bastian, B., Loughnan, S., Haslam, N., \& Radke, H. R. M. (2012). Don’t mind meat? the denial of mind to animals used for human consumption. Personality and Social Psychology Bulletin, 38(2), 247-256. https://doi.org/10.1177/0146167211424291

Berenguer, J. (2010). The Effect of Empathy in Environmental Moral Reasoning. Environment and Behavior, 42, 110-134. https://doi.org/10.1177/0013916508325892

Bilewicz, M., Imhoff, R., \& Drogosz, M. (2011). The humanity of what we eat: Conceptions of human uniqueness among vegetarians and omnivores. European Journal of Social Psychology, 41, 201-209. DOI: 10.1002/ejsp.766

Bloom, P. (2010). How do morals change? Nature, 464, 490. http://dx.doi.org/10.1038/464490a

Buttelmann, D., \& Böhm, R. (2014). The Ontogeny of the Motivation That Underlies InGroup Bias. Psychological Science, 25(4), 921927. _https://doi.org/10.1177\%2F0956797613516802_

Bone, J. (2013). The animal as fourth educator: A literature review of animals and young children in pedagogical relationships. Australian Journal of Early Childhood, 38(2), 57-64. https://doi.org/10.1177/183693911303800208

Bratanova, B., Loughnan, S., \& Bastian, B. (2011). The effect of categorization as food on the perceived moral standing of animals. Appetite, 57(1), 193-196. https://doi.org/10.1016/j.appet.2011.04.020

Busch, J. T., Watson-Jones, R. E., \& Legare, C. H. (2017). The coexistence of natural and supernatural explanations within and across domains and development. The British journal of developmental psychology, 35(1), 4-20. https://doi.org/10.1111/bjdp.12164 
Caviola, L., \& Capraro, V. (2020). Liking but Devaluing Animals: Emotional and Deliberative Paths to Speciesism. Social Psychological and Personality Science, 11(8), 1080-1088. https://doi.org/10.1177\%2F1948550619893959

Caviola, L., Everett, J. A. C., \& Faber, N. S. (2019). The moral standing of animals: Towards a psychology of speciesism. Journal of Personality and Social Psychology, 116(6), 1011-1029. https://doi.org/10.1037/pspp0000182

Christensen, J. , Flexas, A. , Calabrese, M. , Gut, N. , \& Gomila, A. (2014). Moral judgment reloaded: a moral dilemma validation study. Frontiers in Psychology, 5 . https://doi.org/10.3389/fpsyg.2014.00607_

Clement, G. (2013). Animals and Moral Agency: The Recent Debate and Its Implications. Journal of Animal Ethics, 3(1), 1. https://doi.org/10.5406/janimalethics.3.1.0001

Climaterra. (Junio de 2020). El fin de la carne: crecen el vegetarianismo y el veganismo. Climaterra. Recuperado de https://www.climaterra.org/post/el-fin- de-la-carnecrecen-el-vegetarianismo-y-el-veganismo

Costello, K., \& Hodson, G. (2014a). Lay beliefs about the causes of and solutions to dehumanization and prejudice: Do nonexperts recognize the role of human- animal relations? Journal of Applied Social Psychology, 44(4), 278-288. https://doi.org/10.1111/jasp.12221

Costello, K., \& Hodson, G. (2014b). Explaining dehumanization among children: the interspecies model of prejudice. The British journal of social psychology, 53(1), 175-197. https://doi.org/10.1111/bjso.12016

Crimston, D., Bain, P. G., Hornsey, M. J., \& Bastian, B. (2016). Moral expansiveness: Examining variability in the extension of the moral world.

Journal of Personality and Social Psychology, 111(4), 636-653. https://doi.org/10.1037/pspp0000086

Cushman, F. (2015). Deconstructing intent to reconstruct morality. Current Opinion in Psychology, 6, 97-103. https://doi.org/10.1016/j.copsyc.2015.06.003

Cushman, F., Sheketoff, R., Wharton, S., \& Carey, S. (2013). The development of intent- 
Tesis Psicológica | Vol. 17(1) enero-junio /22 | pp. 1-31 | E- ISSN: 2422-045

based moral judgment. Cognition, 127(1), 6-21. https://doi.org/10.1016/j.cognition.2012.11.008_

Dhont, K., \& Hodson, G. (2014). Why do right-wing adherents engage in more animal exploitation and meat consumption? Personality and Individual Differences, 64, 12-17. https://doi.org/10.1016/j.paid.2014.02.002

Dhont, K., Hodson, G., \& Leite, A. C. (2016). Common Ideological Roots of Speciesism and Generalized Ethnic Prejudice: The Social Dominance Human-Animal Relations Model (SD-HARM). European Journal of Personality, 30(6), 507-522. https://doi.org/10.1002/per.2069

DiYanni, C., \& Kelemen, D. (2005). Time to get a new mountain? The role of function in children's conceptions of natural kinds. Cognition, 97(3), 327-335. http://doi.org/10.1016/j.cognition.2004.10.002

Dovidio, J. F., Gaterner, S. L. (2001). Intergroup Bias. In G. Fiske, S., Gilbert, D, Lindzey (Ed.), Handbook of Social Psychology (5th ed., pp. 1084-1121). Wiley

Duhn, I. (2012). Places for pedagogies: Pedagogies for places. Contemporary Issues in Early Childhood, 13(2), 99-106. https://doi.org/10.2304\%2Fciec.2012.13.2.99

El Tiempo (26 de enero de 2018). Cada vez son más los colombianos que tienen mascota. El Tiempo. Recuperado de https://www.eltiempo.com/colombia/cada-vez-son-maslos-colombianos-que- tienen-mascota-175608

Fonseca, M. J., Franco, N. H., Brosseron, F., Tavares, F., Olsson, I. A. S., \& BorlidoSantos, J. (2011). Children's attitudes towards animals: Evidence from the RODENTIA project. Journal of Biological Education, 45(3), 121-128. https://doi.org/10.1080/00219266.2011.576259

Geerdts, M. S., Van de Walle, G. A., \& LoBue, V. (2015). Daily animal exposure and children's biological concepts. Journal of Experimental Child Psychology, 130, 132146. http://doi.org/10.1016/j.jecp.2014.10.001

Gini, G., Pozzoli, T., \& Hymel, S. (2014). Moral disengagement among children and youth: A meta-analytic review of links to aggressive behavior. Aggressive Behavior, 
Tesis Psicológica | Vol. 17(1) enero-junio /22 | pp. 1-31 | E- ISSN: 2422-045

40(1), 56-68. http://doi.org/10.1002/ab.21502

Guevara Benavides L.M. (18 de febrero 2019). Seis de cada 10 hogares del país tienen mascota según Brandstrat. La República. Recuperado de https://www.larepublica.co/consumo/seis-de-cada-10-hogares-del-pais-tienenmascota-segun-brandstrat-2829114

Harris, P. L. (2012). Trusting what you're told: How children learn from others. Cambridge, MA: Belknap Press/Harvard University Press.

Harris, P. L., \& Koenig, M. A. (2006). Trust in testimony: How children learn about science and religion. Child Development, 77, 505-524. https://doi.org/10.1111/j.14678624.2006.00886.x

Heiphetz, L., \& Young, L. (2014). A social cognitive developmental perspective on moral judgment. Behaviour, 151(2-3), 315-335. http://doi.org/10.1163/1568539x-00003131

Hester, N., \& Gray, K. (2020). The Moral Psychology of Raceless, Genderless Strangers. Perspectives on Psychological Science, 15(2), 216-230. https://doi.org/10.1177/1745691619885840

Hussar, K.M. and Harris, P.L. (2010), Children Who Choose Not to Eat Meat: A Study of Early Moral Decision-making. Social Development, 19: 627641. https://doi.org/10.1111/j.1467-9507.2009.00547.x

Hussar, K. M., \& Horvath, J. C. (2011). Do children play fair with mother nature? Understanding children's judgments of environmentally harmful actions. Journal of Environmental Psychology, 31(4), 309-313. https://doi.org/10.1016/j.jenvp.2011.05.001

Janoff-Bulman, R., \& Carnes, N. C. (2013). Surveying the moral landscape: moral motives and group-based moralities. Personality and Social Psychology Review, 17(3), 219-36. http://doi.org/10.1177/1088868313480274

Kahn, P. H. (1997a). Children's moral and ecological reasoning about the Prince William Sound oil spill. Developmental Psychology, Vol. 33, pp. 1091-1096. https://doi.org/10.1037//0012-1649.33.6.1091

Kahn, P. H. (1997b). Developmental Psychology and the Biophilia Hypothesis: 
Tesis Psicológica | Vol. 17(1) enero-junio /22 | pp. 1-31 | E- ISSN: 2422-045

Children's Affiliation with Nature. Developmental Review, 17(1), 1-61. https://doi.org/10.1006/drev.1996.0430

Kahn P. H., Jr (1997). Bayous and jungle rivers: cross-cultural perspectives on children's environmental moral reasoning. New directions for child development, (76), 23-36. https://doi.org/10.1002/cd.23219977604

Kahn, P. H., \& Lourenço, O. (2002). Water, Air, Fire, and Earth: A Developmental Study in Portugal of Environmental Moral Reasoning. Environment and Behavior, 34(4), 405-430. https://doi.org/10.1177/00116502034004001

Kahn, P. H., Severson, R. L., \& Ruckert, J. H. (2009). The human relation with nature and technological nature. Current Directions in Psychological Science, 18(1), 37-42. https://doi.org/10.1111/j.1467-8721.2009.01602.X

Kansky, R. and Knight, A. T. 2014. Key factors driving attitudes towards large mammals in conflict with humans. Biological Conservation 179, 93-105. http://dx.doi.org/10.1016/j.biocon.2014.09.008

Kelemen, D. (1999). The scope of teleological thinking in preschool children.

Cognition, 70(3), 241-272. http://doi.org/10.1016/S0010-0277(99)00010-4 Kelemen, D. (2004). Are children “intuitive theists”? Reasoning about purpose and design in nature. Psychological Science, 15(5), 295-301. http://doi.org/10.1111/j.0956-7976.2004.00672.x

Kortenkamp, K. V., \& Moore, C. F. (2001). Ecocentrism and anthropocentrism: Moral reasoning about ecological commons dilemmas. Journal of Environmental Psychology, 21, 261-272. https://psycnet.apa.org/doi/10.1006/jevp.2001.0205_

Legare, C. H., \& Lombrozo, T. (2014). Selective effects of explanation on learning during early childhood. Journal of Experimental Child Psychology, 126, 198- 212. http://doi.org/10.1016/j.jecp.2014.03.001

Lombrozo, T., \& Carey, S. (2006). Functional explanation and the function of explanation.

$$
\text { Cognition, } \quad \text { 99(2), 167-204. }
$$

http://doi.org/10.1016/j.cognition.2004.12.009 
Tesis Psicológica | Vol. 17(1) enero-junio /22 | pp. 1-31 | E- ISSN: 2422-045

Loughnan, S., Bastian, B., \& Haslam, N. (2014). The Psychology of Eating Animals. Current Directions in Psychological Science, 23(2), 104-108. https://doi.org/10.1177/0963721414525781.

Loughnan, S., Haslam, N., \& Bastian, B. (2010). The role of meat consumption in the denial of moral status and mind to meat animals. Appetite, 55(1), 156-159. https://doi.org/10.1016/j.appet.2010.05.043

Malle, B. F. (2011). Attribution Theories: How People Make Sense of Behavior. En D. Chaade (Ed.), Theories in Social Psychology. (1st ed., pp. 72-95). WileyBlackwell

Malle, B. F., Guglielmo, S., \& Monroe, A. E. (2014). A Theory of Blame. Psychological Inquiry, 25(2), 147-186. http://doi.org/10.1080/1047840X.2014.877340

Melo Cortés T. (17 de junio de 2019). Hay más de 600 millones de vegetarianos en el mundo. RCN Radio. Recuperado de https://www.rcnradio.com/estilo-de- vida/haymas-de-600-millones-de-vegetarianos-en-el-mundo

Melson, G. F. (2013). Children's ideas about the moral standing and social welfare of nonhuman species. Journal of Sociology and Social Welfare, 40(4), 81- 106.

Melson, G. F., \& Fine, A. H. (2010). Animals in the Lives of Children. In A. H. Fine (Ed.), Handbook on Animal-Assisted Therapy (Third Edit, Vol. 2008, pp. 223- 245). https://doi.org/10.1016/B978-0-12-381453-1.10012-1.

Melson, G. F., Kahn, P. H., Beck, A., Friedman, B., Roberts, T., Garrett, E., \& Gill, B. T. (2009). Children's behavior toward and understanding of robotic and living dogs. Journal of Applied Developmental Psychology, 30(2), 92-102. https://doi.org/10.1016/j.appdev.2008.10.011

Miralles, A., Raymond, M., \& Lecointre, G. (2019). Empathy and compassion toward other species decrease with evolutionary divergence time. Scientific reports, 9(1), 19555. https://doi.org/10.1038/s41598-019-56006-9

Mercier, H. (2011). What good is moral reasoning? Mind \& Society, 10(2), 131-148 https://doi.org/10.1007/s11299-011-0085-6 
Tesis Psicológica | Vol. 17(1) enero-junio /22 | pp. 1-31 | E- ISSN: 2422-045

Moore, C. (2015). Moral disengagement. Current Opinion in Psychology, 6, 199- 204. http://doi.org/10.1016/j.copsyc.2015.07.018

Moreno-Romero, C.O. (2020). Desentendimiento moral y atribución de culpa: Encuentros y desencuentros en el estudio de la cognición moral. Revista $\begin{array}{llll}\text { Colombiana } \quad \text { de } & \text { Psicología, } & \text { 29, }\end{array}$ https://doi.org/10.15446/rcp.v29n1.76536

Myers, O.E., Jr. (2007). The significance of children and animals: Social development and our connection to other species (rev. ed.). West Lafayette, IN: Purdue University Press.

Neldner K, Crimston C, Wilks M, Redshaw J, Nielsen M. (2018) The developmental origins of moral concern: An examination of moral boundary decision making $\begin{array}{llll}\text { throughout } & \text { childhood. } & \text { ONE } & \text { 13(5) }\end{array}$ https://doi.org/10.1371/journal.pone.0197819

Nucci, L., \& Turiel, E. (2009). Capturing the complexity of moral development and education. Mind, brain, and education, 3(3), 151-

159. https://doi.org/10.1111/j.1751-228X.2009.01065.X

Obermann, M.-L. (2013). Temporal Aspects of Moral Disengagement in School Bullying: Crystallization or Escalation? Journal of School Violence, 12(January 2015), 193210. http://doi.org/10.1080/15388220.2013.766133

Olthof, T., Rieffe, C., Terwogt, M. M., Lalay-Cederburg, C., Reijntjes, A., \& Hagenaar, J. (2008). The assignment of moral status: Age-related differences in the use of three mental capacity criteria. British Journal of Developmental Psychology, 26(2), 233247. https://doi.org/10.1348/026151007X216036

Overton, W. (2013). A new paradigm for developmental science: Relationism and relational- developmental systems. Applied Developmental Science, 17, 94- 107. https://doi.org/10.1080/10888691.2013.778717

Piaget, J. (1965/1932). The moral judgment of the child. New York: Free Press.

Piazza, J., \& Loughnan, S. (2016). When Meat Gets Personal, Animals’ Minds Matter Less: Motivated Use of Intelligence Information in Judgments of Moral Standing. 
Tesis Psicológica | Vol. 17(1) enero-junio /22 | pp. 1-31 | E- ISSN: 2422-045

Social Psychological and Personality Science, 7(8), 867-874. https://doi.org/10.1177/1948550616660159

Pinker, S. (2011). The better angels of our nature: Why violence has declined. London, England: Penguin.

Pozzoli, T., Gini, G., \& Vieno, A. (2012). Individual and Class Moral Disengagement in Bullying Among Elementary School Children. Aggressive Behavior, 38(5), 378388. http://doi.org/10.1002/ab.21442

Rhodes, M., Leslie, S. J., \& Tworek, C. M. (2012). Cultural transmission of social essentialism. Proceedings of the National Academy of Sciences of the United States of America, 109(34), 13526-13531. https://doi.org/10.1073/pnas.1208951109

Rosch, E. (1999). Principles of categorization. In E. Margolis \& S. Laurence (Eds.), Concepts: Core readings (pp. 189-206). Cambridge, MA: MIT Press Rothgerber, H.

(2013). Real men don't eat (vegetable) quiche: Masculinity and the justification of meat consumption. Psychology of Men and Masculinity, 14(4), 363375. https://doi.org/10.1037/a0030379

Rozin, P. (2007). Food and eating. In S. Kitayama \& D. Cohen (Eds.), The handbook of cultural psychology (pp. 391-416). New York, NY: The Guilford Press.

Ruby, M. B., \& Heine, S. J. (2011). Meat, morals, and masculinity. Appetite, 56(2), 447450. https://doi.org/10.1016/j.appet.2011.01.018

Ruby,M. B. (2012). Vegetarianism. A blossoming field of study. Appetite, 58, 141- 150. http://dx.doi.org/10.1016/j.appet.2011.09.019

Ruckert, J. H. (2016). Justice For All ? Children's Moral Reasoning about the Welfare and Rights of Endangered Species Justice For All ? Children's Moral Reasoning about the Welfare and Rights of Endangered Species. 7936(June). https://doi.org/10.1080/08927936.2015.1093297

Sanbonmatsu, J. (2014). The animal of bad faith: Speciesism as an existential project. Critical Animal Studies: Thinking the Unthinkable, (June), 29-45. Recuperado de https://www.researchgate.net/profile/John Sanbonmatsu/publication/2794491 
Tesis Psicológica | Vol. 17(1) enero-junio /22 | pp. 1-31 | E- ISSN: 2422-045

14 The Animal of Bad Faith Speciesism as an Existential Project/links/5 592f2a908ae5af2b0eb65a7.pdf

Schein, C. (2020). The Importance of Context in Moral Judgments. Perspectives on Psychological Science, 15(2), 207-215. https://doi.org/10.1177/1745691620904083

Severson, R. L., \& Kahn, P. H. (2010). In the orchard: Farm worker children's moral and environmental reasoning. Journal of Applied Developmental Psychology, 31(3), 249-256. https://doi.org/10.1016/j.appdev.2010.02.003

Severson, R. L., \& Lemm, K. M. (2016). Kids see human too: Adapting an individual differences measure of anthropomorphism for a child sample. Journal of Cognition and Development, 17, 122-141. https://doi.org/10.1080/15248372.2014.989445

Sevillano, V., \& Fiske, S. T. (2016). Warmth and competence in animals. Journal of Applied Social Psychology, 46, 276-293. https://doi.org/10.1111/jasp.12361

Sevillano, V., \& Fiske, S. T. (2019). Stereotypes, emotions, and behaviors associated with animals: A causal test of the stereotype content model and BIAS map. Group Processes and Intergroup Relations, 22(6), 879-900. https://doi.org/10.1177/1368430219851560

Sloman, S. A., \& Lagnado, D. (2015). Causality in Thought. Annual Review of Psychology, 66(1), 223-247. https://doi.org/10.1146/annurev-psych-010814-015135

Smetana, J. G., Jambon, M., Conry-Murray, C., \& Sturge-Apple, M. L. (2012). Reciprocal Associations between Young Children's Developing Moral Judgments and Theory of Mind. Developmental Psychology, 48(4), 1144- 1155. https://psycnet.apa.org/doi/10.1037/a0025891

Sommer, K., Nielsen, M., Draheim, M., Redshaw, J., Vanman, E. J., \& Wilks, M. (2019). Children's perceptions of the moral worth of live agents, robots, and inanimate objects. Journal of Experimental Child Psychology, 187, 104656. https://doi.org/10.1016/j.jecp.2019.06.009_

Šorytė, D., \& Pakalniškienè, V. (2019). Why it is important to protect the environment: reasons given by children. International Research in Geographical and 
Tesis Psicológica | Vol. 17(1) enero-junio /22 | pp. 1-31 | E- ISSN: 2422-045

Environmental Education, 28(3), 228-241. https://doi.org/10.1080/10382046.2019.1582771

Timm, S. C. (2016). Moral Intuition or Moral Disengagement? Cognitive Science Weighs in on the Animal Ethics Debate. Neuroethics, 9(3), 225-234. https://doi.org/10.1007/s12152-016-9271-x

Topolski, J. R., Weaver, N., Martin, Z., \& McCoy J. (2013) Choosing between the Emotional Dog and the Rational Pal: A Moral Dilemma with a Tail, Anthrozoös: A multidisciplinary journal of the interactions of people and animals, 26:2, 253- 263 http://www.tandfonline.com/loi/rfan20

Townsend, D., Taylor, L. K., Merrilees, C. E., et al. (2020). Youth in Northern Ireland: Linking Violence Exposure, Emotional Insecurity, and the Political Macrosystem. Monographs of the Society for Research in Child Development, 85(4). https://doi.org/10.1111/mono.12423.

Uskul, A. K., \& Oishi, S. (2020). Editorial overview: What is socio-ecological psychology? Current Opinion in Psychology, 32, 181-184. https://doi.org/10.1016/j.copsyc.2020.01.001

Waytz, A., Cacioppo, J., \& Epley, N. (2010). Who Sees Human?: The Stability and Importance of Individual Differences in Anthropomorphism. Perspectives on Psychological Science, 5(3), 219 -

232. https://doi.org/10.1177/1745691610369336

Waytz, A., Gray, K., Epley, N., \& Wegner, D. M. (2010). Causes and consequences of mind perception. Trends in Cognitive Science, 14, 383-388. https://doi.org/10.1016/j.tics.2010.05.006

Wilks, M., Caviola, L., Kahane, G., \& Bloom, P. (2021). Children Prioritize Humans Over Animals Less Than Adults Do. Psychological Science, 32(1), 27-38. https://doi.org/10.1177/0956797620960398

Zhang, Z., Grocke, P., \& Tomasello, M. (2019). The influence of intention and outcome on young children's reciprocal sharing. Journal of experimental child psychology, 187, 104645. https://doi.org/10.1016/j.jecp.2019.05.012 